\title{
Energy expenditure: how much does it matter in infant and pediatric chronic disorders?
}

\author{
Nilesh M. Mehta'
}

\begin{abstract}
A sound understanding of energy needs during chronic illness is necessary to avoid imbalances in energy intake and requirements. Failure to accurately estimate energy needs results in both underfeeding and overfeeding in chronically ill children. Suboptimal energy and protein intake may lead to deterioration in body composition, particularly lean body mass loss, which eventually impacts functional outcomes in these vulnerable groups. Furthermore, infants and children with chronic illnesses have a high prevalence of malnutrition and can ill afford further nutritional deterioration from suboptimal nutrient delivery. On the other hand, unintended delivery of excessive energy in patients with chronic respiratory insufficiency results in increased respiratory burden and poor outcomes. Hence, awareness of the energy requirements and attention to energy and protein balance are important when caring for children with chronic illnesses. The basic concepts of the metabolic stress response, measurement of energy expenditure, and the impact of energy imbalance on clinical outcomes in children with chronic illness are reviewed.
\end{abstract}

$\mathbf{T}$ he prediction of energy requirement during illness is challenging. The energy burden is variable and may be dependent on the type, severity, and stage of illness. Failure to accurately estimate energy expenditure leads to erroneous energy prescription and results in unintended energy imbalance. The inability to deliver the prescribed nutrients during acute or chronic illness further contributes to the mismatch between energy requirements and intake. Energy imbalance is associated with poor outcomes. Energy imbalance may be particularly relevant to infants and children with existing malnutrition or obesity, in whom further nutritional deterioration due to suboptimal energy delivery is not desirable.

Chronic illness is a condition, disease, or disorder that is characterized by long duration and, in some cases, slow progression, which may be associated with episodic periods of worsening. Although there is no clear definition or cutoff for defining chronic illness in children, prolonged incapacitation is likely to place significant nutritive challenges and risk of energy imbalance. For the purpose of this review, the focus is on children with illnesses that require care in the community or hospital setting for more than $2 \mathrm{wk}$. Failure to meet the nutritional needs of patients with chronic illness may be associated with higher morbidity and mortality. In recent years, there has been an increase in awareness of overfeeding, in which energy delivery is much higher than the requirements. This is due to the overestimation of energy needs, either due to inaccuracy of the common equations used for this purpose or due to the use of stress factors based on presumed hypermetabolism. On the other hand, energy expenditure during certain chronic illnesses or during the rehabilitative phase after an acute illness may be characterized by prolonged hypermetabolism and increased energy expenditure. Failure to meet energy needs in this subpopulation could result in cumulative energy deficits. Hence, both underfeeding and overfeeding must be avoided in the chronically ill child. A sound understanding of the metabolic response to illness, accurate estimation of energy requirements, attention to the delivery of prescribed nutrients, and awareness of cumulative energy balance are necessary to ensure optimal nutritional support in chronically ill infants and children.

\section{METABOLIC RESPONSE TO STRESS}

The human response to the stress of injury, illness, or surgery is stereotypical and involves a series of metabolic changes (1). This metabolic response is driven by a complex neuroendocrine system and may be correlated to the nature and severity of the insult. In its original description, the response was characterized as biphasic, with brief ebb phase followed by a hypermetabolic flow phase (1). This hypermetabolic phase is catabolic in nature. It is driven initially by a cytokine surge and increased counter regulatory hormones with insulin and growth hormone resistance. The result is breakdown of endogenous body stores, in particular muscle mass, to provide free amino acids that are used for the inflammatory response, tissue repair, and wound healing (2). This is an adaptive phenomenon where autocannibalism sustains the individual during periods of low nutrient availability after the insult or injury.

Recent accounts of measured energy expenditure have shown a muted hypermetabolic response after major illness, injury, or surgery. One exception is burn injury, which is characterized by a profound hypermetabolic response that may be sustained for several weeks (3). By contrast, metabolic 
measurements in most other illnesses reveal a muted and brief hypermetabolic response, compared with past descriptions. Other factors that impact the nature of the stress response include nutritional status, endogenous metabolic reserve, and interventions in the postinjury period. In the chronically ill child or one with protracted acute illness, the hypermetabolic response is expected to abate, and energy expenditure returns to baseline. Furthermore, the energy expenditure incurred by the stress response may be variable throughout the course of chronic illnesses, especially in illnesses that are characterized by episodic flares, worsening, or intercurrent complications.

Hence, a careful approach to accurately determine energy needs during chronic illnesses is essential. Both underestimation and overestimation of energy needs during protracted illness may impact the ability to match the requirements with intake and result in unintended energy imbalance. With the advent of newer technology, there are an increasing number of portable devices for measuring energy expenditure and monitoring physical activity. These devices now use computerized systems to track energy balance, and could facilitate optimal nutritional therapy in vulnerable children with chronic illnesses.

\section{MEASUREMENT OF RESTING ENERGY EXPENDITURE}

Indirect calorimetry (IC) has been used to determine energy expenditure in hospitalized children for several decades. It has emerged as a gold-standard method for assessment of energy needs and helps guide optimal energy prescription (4). A review of the basics of metabolic monitoring by IC, currently available devices, and challenges to measuring energy expenditure in children has been recently published (5). However, IC is not widely available and hence only utilized in few centers where the necessary resources and expertise are available. Even in centers with available resources, IC is often used for research rather than regular clinical applications. Children with chronic illnesses who are cared for in the home setting are particularly deprived of IC testing, as portable technology is required to obtain metabolic assessments. In the absence of IC, a variety of equations have been developed and employed as a surrogate method to estimate energy expenditure. These equations are based on demographic and anthropometric variables such as age, sex, weight, and height (6). Most equations used to estimate resting energy expenditure (REE) were derived from healthy population data (7). Hence, standard equations have a high likelihood of inaccuracy in determining accurate REE in sick patients. There have been similar reports of inaccuracy of these equations in children with chronic illnesses (8-10). Shakur et al. compared predicted REE by a standard equation and measured REE by IC, in 398 chronically ill children admitted to a tertiary referral hospital. Their cohort included children with growth failure, cystic fibrosis (CF), short bowel syndrome, liver disease, cerebral palsy, inflammatory bowel disease, eating disorder, developmental delay, and genetic disorders. Resting energy expenditure was predicted using the equation, and measured resting energy expenditure (MEE) was determined by IC. The use of the Schofield equation estimate to prescribe daily energy intake would have resulted in over half their cohort to be underfed due to underestimation of the REE. There was a poor agreement between the measured and predicted REE with a mean bias (limits) of $42(-387.9,474.3)$ $\mathrm{kcal} / \mathrm{d}$. These wide limits suggest a potential for both underestimation and overestimation of REE by the equation. In a cohort of children with acute and chronic illnesses, we have reported the likelihood of overestimating energy expenditure by the Schofield, Harris-Benedict, and the World Health Organization equations (11). Bechard et al. reported REE measurements in 26 children at baseline and $30 \mathrm{~d}$ following hemopoietic stem cell transplantation. A significant decrease in REE was noted with a nadir at around $7 \mathrm{~d}$ after hemopoietic stem cell transplantation (12). During the 30-d study period, REE was much lower than expected, and the subjects, who were all exclusively dependent of parenteral nutrition, were at a high risk of overfeeding. On the other hand, children with burn injury have been reported to be hypermetabolic with increased energy expenditure for up to many months after the insult (13). In some chronic illnesses, the impact of energy expenditure on nutritional outcomes is not clear. Bott et al. (14) described measured REE in a group of children with chronic lung disease. In this study, airway obstruction was not related to REE, and the authors concluded that growth failure was probably a long-term fixed consequence of the bronchopulmonary dysplasia in infancy. Unintended underfeeding and overfeeding are associated with negative outcomes and must be prevented. Increased awareness of energy balance and careful determination of energy expenditure will help optimize energy intake and balance.

In a recent study of children with chronic respiratory failure, we explored a unique home-based model of individualized nutritional prescription for this cohort that was dependent on long-term respiratory support at home (15). We recorded a high prevalence of malnutrition, suboptimal energy intake, and altered metabolic state in this group. We measured REE in these subjects and examined the agreement with predicted energy expenditure by the Schofield weight-based equations. We recorded poor agreement between the estimated and

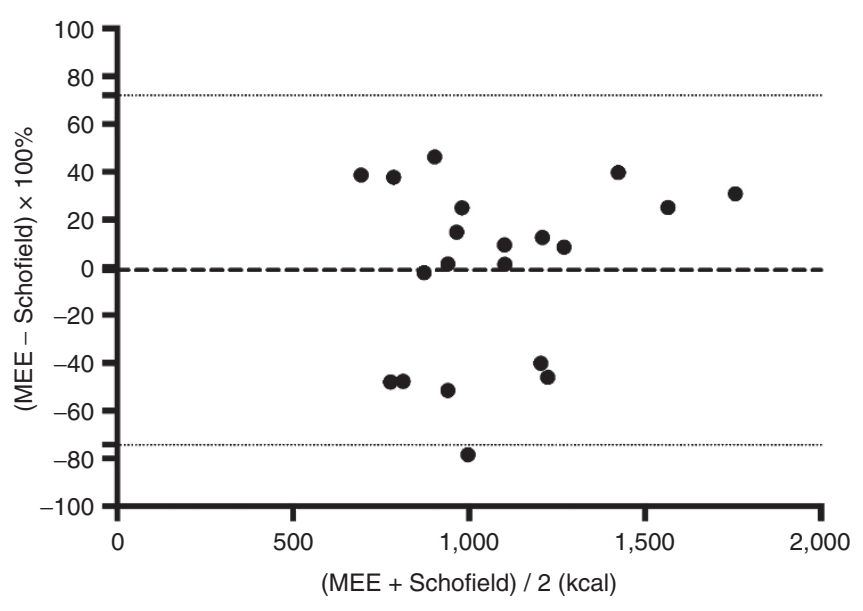

Figure 1. Bland-Altman plot showing agreement between measured resting energy expenditure (MEE) by indirect calorimetry and predicted REE by the Schofield equations. Mean bias for agreement was $-1.31 \%$ with limits $(-74.3$ to $+72 \%)$. REE, resting energy expenditure. 
measured values for energy expenditure, with a mean bias of $-1.31 \%$ and wide limits $(-74.3$ to $+72 \%)$. Figure 1 shows the Bland-Altman plot for agreement between the two methods in this study. The use of equation-estimated energy expenditure values in this cohort would have placed the vulnerable children at risk of cumulative positive or negative energy balance. An individualized approach where energy intake is guided by accurate assessments of energy expenditure allowed nutrient intake to be optimized based on measured REE values in this cohort. The nutritional intervention in this study, 12 wk of individualized prescription, was associated with reductions in carbon dioxide production and minute ventilation in this group (Martinez EE, personal communication). This study illustrates the importance of energy expenditure assessment in children with chronic illnesses and its potential impact on clinical outcomes. IC requires resources and is not widely available. In order to explore the concept of individualized nutrition in the wider chronically ill population, other modes of energy expenditure assessment are required.

\section{MONITORING PHYSICAL ACTIVITY DURING CHRONIC ILLNESS}

Figure 2 shows the components of total energy expenditure (TEE). Basal metabolic rate is the principal component of TEE. Diet-induced thermogenesis represents the cost of food assimilation and processing, accounting for $\sim 10 \%$ of the TEE in children receiving enteral bolus feeds (16). The other important component is physical activity, which may contribute from 15 to $40 \%$ of the TEE (17). Chronically ill children may have varied physical activity profiles, ranging from bedridden, sedentary to overactive. Lack of physical activity in children with chronic illness may be due to the underlying disorder (juvenile rheumatoid arthritis), deconditioning from prolonged critical illness, or secondary to cumulative nutritional deficiencies (from decreased intake, poor absorption, or increased losses of nutrients). On the other hand, certain

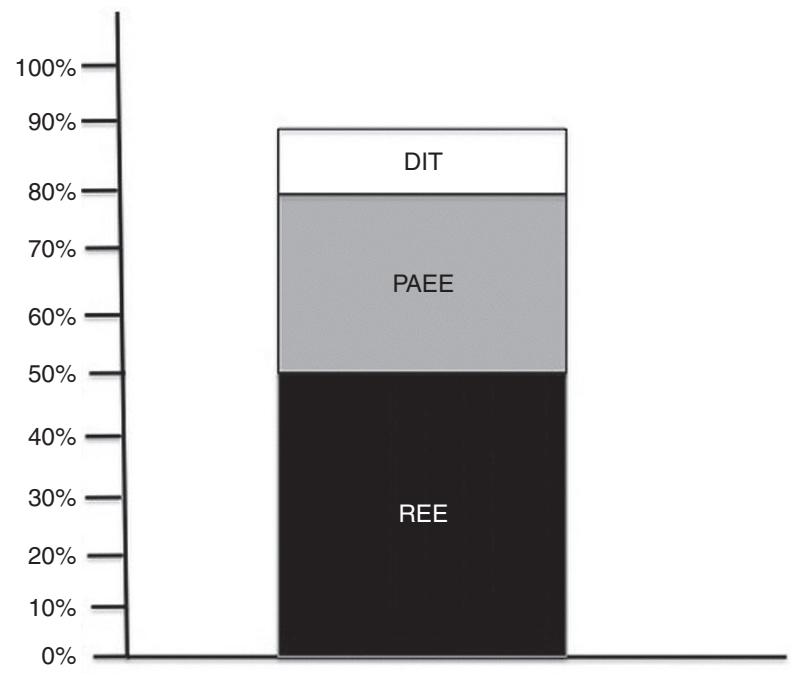

Figure 2. Components of total energy expenditure in a child (13). DIT, diet-induced thermogenesis; PAEE, physical activity energy expenditure; $\mathrm{REE}$, resting energy expenditure. Reprinted with permission from ref. (13). conditions may be associated with increased physical activity (both exercise and nonexercise related) such as poorly controlled seizure disorder, hyperactivity associated with some diseases, and dysautonomia. Furthermore, the amount of physical activity may increase over the course of illness in previously sedentary patients. Hence, determination of energy expenditure related to physical activity is crucial when prescribing daily nutrition goals.

A variety of methods have been used to record physical activity in chronically ill patients. These are generally classified into three levels: (i) direct observation, (ii) secondary methods, and (iii) subjective measures. Direct observation remains the gold standard for assessing the energy burden from physical activity. Subjective measures include surveys that elicit responses that help estimate physical activity. Although they are easy to perform and relatively inexpensive, their correlation with direct observed activity or measured energy expenditure may be variable and prone to errors. With the advent of technology, secondary objective methods such as portable heart rate monitors, pedometers, and accelerometers are now increasingly available and have been incorporated into the estimation of TEE in chronically ill patients (18). A combination of accelerometer and heart rate has been shown to provide accurate prediction of the physical activity energy expenditure (PAEE) in children $(19,20)$. Accelerometers use piezoelectric transducers and microprocessors that convert acceleration produced by body movements into a digital signal. In a recent study by Takken et al. (19), a chest mounted, combined-unit was employed in a Dutch cohort of children aged 8 to $18 \mathrm{y}$, for synchronized heart rate and accelerometer recordings. These variables were then used to derive PAEE using a previously validated formula (20). Bland-Altman analysis of agreement between the derived PAEE value and that obtained by IC (MREE after activity minus MREE during rest) revealed a mean bias of 2.1, but the limits of agreement were wide $(+148$ to -141 ; Figure 3 ). The authors concluded that monitors of heart rate and motion might be valid for determination of activity energy expenditure in groups of children with chronic

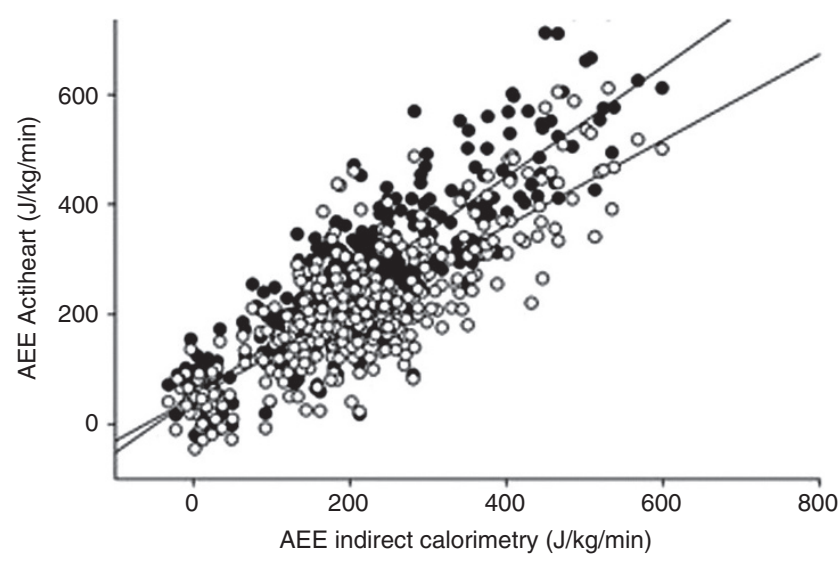

Figure 3. Scatter plot of actual AEE as measured by indirect calorimetry and estimated by two Actiheart prediction equations, used in the Corder et al. (20) study (dark circles) and the Takken et al. (19) study (empty circles). AEE, activity energy expenditure. Reprinted with permission from ref. (19). 


\section{Energy epenentiture inctronoicillines $\mid$ Review}

illnesses. Their use in individual patients may be erroneous and should be interpreted with caution. Future research must explore the validity of the next generation of motion sensors in pediatric populations.

\section{ENERGY IMBALANCE DURING CHRONIC ILLNESS AND ITS IMPACT ON OUTCOMES}

Accurate assessment of TEE in chronic illness facilitates the goal of optimizing energy prescription in this group. The metabolic response to chronic illness may be variable and unpredictable, leading to inaccuracy of equations used to predict energy expenditure and consequentially a high probability of unintended underfeeding or overfeeding of chronically ill children. The resultant energy imbalance may accumulate over time, with deterioration of nutritional status and negative impact on patient outcomes. Beyond the simplistic paradigm of net balance as a difference between energy intake and expenditure, there may be adaptive changes between the various components of TEE and behavioral changes that might influence energy balance during protracted illness (16). In patients with $\mathrm{CF}$, the increased energy expenditure from disease-associated hypermetabolism is often offset by simultaneous reduction in PAEE with no net increase in TEE (21). Furthermore, there may be inaccurate reporting of nutrient intake that contributes to erroneous energy balance calculations in some chronic illnesses. In children with CF, parental report of the child's energy intake may be higher than the actual amount (22). Trabulsi et al. observed overreporting of actual energy intake in a quarter of their cohort of preadolescent children with CF. On the other hand, in adolescents with obesity, daily energy intake may be underreported (23). This underreporting or overreporting of energy intake impacts the accuracy of energy balance calculations and makes it difficult to study chronically ill subjects outside the supervised environment of the hospital.

Unintended underfeeding could be potentially harmful in chronically ill children with preexisting malnutrition. Cumulative energy deficits are associated with loss of muscle mass, poor wound healing, increased risk of infections, increased morbidity, and a higher risk of mortality in critically ill adults $(24,25)$. Both energy and protein deficits are undesirable. Protein deficits have been associated with nutritional deterioration in critically ill infants and children (26). Achievement of protein delivery goals is associated with decreased mortality in mechanically ventilated adults (27). In critically ill children, we have recently shown a decrease in odds of 60-d mortality associated with increased energy intake adequacy (28). In this multicenter study of over 500 mechanically ventilated children, energy adequacy (percentage of prescribed energy that was delivered) of $66 \%$ compared with $33 \%$ was associated with lower mortality (odds ratio: $0.27 ; P<$ 0.002 ). Hence, energy deficits must be prevented, especially in the vulnerable patient with existing malnutrition.

On the other hand, unintended overfeeding due to overestimation of energy needs is also associated with negative consequences. Occult overfeeding is probably prevalent in children with chronic illnesses. Excess of energy from carbohydrate sources increases carbon dioxide production $\left(\mathrm{VCO}_{2}\right)$ and may worsen respiratory insufficiency in patients with chronic pulmonary illness. In a double-blind randomized crossover study in adults with chronic obstructive lung disease, consumption of a high-energy drink was associated with increased $\mathrm{VCO}_{2}$ and deterioration of performance in a 6-min walk test, compared with a noncalorific control drink (29). The relationship between overfeeding and $\mathrm{VCO}_{2}$ was explored by Talpers et al. (30) in stable mechanically ventilated adults. Excess of caloric feeding was associated with increased $\mathrm{VCO}_{2}$ in this study. The percentage of carbohydrate in the diet did not increase $\mathrm{VCO}_{2}$ as long as caloric intake was in moderation. Overfeeding has also been associated with increased risk of infection, hyperglycemia, fatty liver, and increased metabolic demand for storage of surplus of energy substrates. However, during the rehabilitative phase of chronic illnesses, children may experience a growth spurt. In such cases, energy intake may be higher than REE in order to account for the allowance for growth.

\section{CONCLUSIONS}

Energy burden of the human metabolic response to stress is variable and cannot be easily predicted. In children with chronic illnesses, standard equations may be inaccurate and feeding strategies based on these equations might result in unintended underfeeding or overfeeding and energy imbalance. Cumulative effects of energy imbalance can negatively impact patient outcomes and must be prevented. Accurate assessment of energy expenditure will help optimize energy intake during chronic illnesses. Recent advances in portable IC and the availability of heart rate monitors and motion-sensing devices will allow assessment of PAEE and accurate estimation of TEE. An individualized approach to nutrient delivery in chronically ill children, with judicious energy prescription, attention to delivery of prescribed nutrients, and increased awareness of energy needs, will help avoid both underfeeding and overfeeding, with the potential for improving clinical outcomes.

\section{STATEMENT OF FINANCIAL SUPPORT}

No financial assistance was received to support this article.

\section{REFERENCES}

1. Cuthbertson D. Intensive-care-metabolic response to injury. Br J Surg 1970;57:718-21.

2. Mehta NM, Duggan CP. Nutritional deficiencies during critical illness. Pediatr Clin North Am 2009;56:1143-60.

3. Herndon DN, Curreri PW. Metabolic response to thermal injury and its nutritional support. Cutis 1978;22:501-6, 514.

4. Mehta NM, Compher C; A.S.P.E.N. Board of Directors. A.S.P.E.N. Clinical Guidelines: nutrition support of the critically ill child. JPEN J Parenter Enteral Nutr 2009;33:260-76.

5. Mehta NM, Smallwood CD, Graham RJ. Current applications of metabolic monitoring in the pediatric intensive care unit. Nutr Clin Pract 2014;29:338-47.

6. Sion-Sarid R, Cohen J, Houri Z, Singer P. Indirect calorimetry: a guide for optimizing nutritional support in the critically ill child. Nutrition 2013;29:1094-9.

7. Schofield WN. Predicting basal metabolic rate, new standards and review of previous work. Hum Nutr Clin Nutr 1985;39:Suppl 1:5-41. 
8. Hardy CM, Dwyer J, Snelling LK, Dallal GE, Adelson JW. Pitfalls in predicting resting energy requirements in critically ill children: a comparison of predictive methods to indirect calorimetry. Nutr Clin Pract 2002;17:182-9.

9. Sentongo TA, Tershakovec AM, Mascarenhas MR, Watson MH, Stallings VA. Resting energy expenditure and prediction equations in young children with failure to thrive. J Pediatr 2000;136:345-50.

10. Shakur YA, Richards H, Pencharz PB. Is it necessary to measure resting energy expenditure in clinical practice in children? J Pediatr 2008; 152:437-9.

11. Mehta NM, Bechard LJ, Dolan M, Ariagno K, Jiang H, Duggan C. Energy imbalance and the risk of overfeeding in critically ill children. Pediatr Crit Care Med 2011:12:398-405.

12. Bechard LJ, Feldman HA, Venick R, et al. Attenuation of resting energy expenditure following hematopoietic SCT in children. Bone Marrow Transplant 2012;47:1301-6.

13. Williams FN, Herndon DN, Jeschke MG. The hypermetabolic response to burn injury and interventions to modify this response. Clin Plast Surg 2009;36:583-96.

14. Bott L, Béghin L, Hankard R, Pierrat V, Gondon E, Gottrand F. Resting energy expenditure in children with neonatal chronic lung disease and obstruction of the airways. Br J Nutr 2007;98:796-801.

15. Martinez EE, Smallwood CD, Bechard LJ, Graham RJ, Mehta NM. Metabolic assessment and individualized nutrition in children dependent on mechanical ventilation at home. J Pediatr 2014, in press.

16. Reilly JJ. Energy balance and its measurement in childhood disease. Pediatr Blood Cancer 2008;50:Suppl 2:452-5; discussion 468.

17. Reilly JJ, Edwards CA, Weaver LT. Malnutrition in children with cystic fibrosis: the energy-balance equation. J Pediatr Gastroenterol Nutr 1997;25:127-36.

18. Sirard JR, Pate RR. Physical activity assessment in children and adolescents. Sports Med 2001;31:439-54.

19. Takken T, Stephens S, Balemans A, et al. Validation of the Actiheart activity monitor for measurement of activity energy expenditure in children and adolescents with chronic disease. Eur J Clin Nutr 2010; 64:1494-500.

20. Corder K, Brage S, Mattocks C, et al. Comparison of two methods to assess PAEE during six activities in children. Med Sci Sports Exerc 2007;39:2180-8.

21. Reilly JJ. Understanding chronic malnutrition in childhood and old age: role of energy balance research. Proc Nutr Soc 2002;61:321-7.

22. Trabulsi J, Schall JI, Ittenbach RF, et al. Energy balance and the accuracy of reported energy intake in preadolescent children with cystic fibrosis. Am J Clin Nutr 2006;84:523-30.

23. Reilly JJ, Ness AR, Sherriff A. Epidemiological and physiological approaches to understanding the etiology of pediatric obesity: finding the needle in the haystack. Pediatr Res 2007;61:646-52.

24. Bartlett RH, Dechert RE, Mault JR, Ferguson SK, Kaiser AM, Erlandson EE. Measurement of metabolism in multiple organ failure. Surgery 1982;92:771-9.

25. Faisy C, Lerolle N, Dachraoui F, et al. Impact of energy deficit calculated by a predictive method on outcome in medical patients requiring prolonged acute mechanical ventilation. Br J Nutr 2009;101:1079-87.

26. Hulst J, Joosten K, Zimmermann L, et al. Malnutrition in critically ill children: from admission to 6 months after discharge. Clin Nutr 2004; 23:223-32.

27. Weijs PJ, Stapel SN, de Groot SD, et al. Optimal protein and energy nutrition decreases mortality in mechanically ventilated, critically ill patients: a prospective observational cohort study. JPEN J Parenter Enteral Nutr 2012;36:60-8.

28. Mehta NM, Bechard LJ, Cahill N, et al. Nutritional practices and their relationship to clinical outcomes in critically ill children-an international multicenter cohort study ${ }^{\star}$. Crit Care Med 2012;40:2204-11.

29. Efthimiou J, Mounsey PJ, Benson DN, Madgwick R, Coles SJ, Benson MK. Effect of carbohydrate rich versus fat rich loads on gas exchange and walking performance in patients with chronic obstructive lung disease. Thorax 1992;47:451-6.

30. Talpers SS, Romberger DJ, Bunce SB, Pingleton SK. Nutritionally associated increased carbon dioxide production. Excess total calories vs high proportion of carbohydrate calories. Chest 1992;102:551-5. 